\title{
Zum Tod von Andreas Pfitzmann
}

Am 23.09.2010 ist zu unserer großen Bestürzung im Alter von erst 52 Jahren überraschend Professor Dr. Andreas Pfitzmann verstorben. Als Mitglied des DuD-Beirats hat er die Entwicklung unserer Zeitschrift über mehr als zwei Jahrzehnte begleitet.

Bereits Mitte der 80er Jahre hatte Andreas Pfitzmann mit seinen Forschungsarbeiten an der Universität Karlsruhe den "technischen Datenschutz" begründet. In seiner Dissertation schlug er vor über 20 Jahren Protokolle zur Realisierung teilnehmerüberprüfbaren Datenschutzes für das Dienste integrierende Datennetz ISDN aus Datenschutzsicht fast eine Art "konzeptionelle Blaupause" des heutigen Internet - vor, darunter zahlreiche Ansätze, die wir heute als „Privacy Enhancing Technologies" kennen. Viele dieser Konzepte realisierte er zusammen mit Studenten und Mitarbeitern zunächst in Karlsruhe, später an den Universitäten Hildesheim und Dresden in Laborumgebungen; einige, wie der auf Mix-Netzen basierende und im Rahmen des AN.ON-Projekts entwickelte Web-Anonymizer JAP (heute JonDo), reiften zum Produkt.

Andreas Pfitzmann war ein einflussreicher Mahner und Mittler zwischen den Welten der Informatik, des Rechts und gesellschaftlicher Fragestellungen. Er hat durch seine mehr als 250 Veröffentlichungen und Stellungnahmen wichtige Weichenstellungen der Technikgestaltung beeinflusst, unter anderem als Gutachter des Bundesverfassungsgerichts zu Online-Durchsuchung und Vorratsdatenspeicherung, sowie als Mitautor des zusammen mit den Professoren Hansjürgen Garstka und Alexander Roßnagel 2001 verfassten und viel zitierten Gutachtens zur "Modernisierung des Datenschutzrechts" für das Bundesministerium des Inneren. Auch als langjähriger Vorstand der Fachgruppe "Verlässliche IT-Systeme" und Mitglied des Präsidiumsarbeitskreises „Datenschutz und IT-Sicherheit" der Gesellschaft für Informatik hat Andreas Pfitzmann wiederholt (fach-) politisch Position bezogen - zunächst in der "Kryptodebatte" gegen das Verbot starker Verschlüsselungsverfahren, gegen staatliche Schlüsselhinterlegungen und später gegen die Vorratsdatenspeicherung von Telekommunikationsdaten.

Aber er war auch mit ganzem Herzen Hochschullehrer. Hunderte Studenten begeisterte er für Datenschutz und Datensicherheit, und viele sind dem Thema beruflich treu geblieben, einige inzwischen selbst als Lehrstuhlinhaber.

Nicht überall stießen seine Einschätzungen und Bewertungen auf ungeteilte Zustimmung. Das ist unvermeidlich für jemanden, der für seine Überzeugungen streitet. Seine Fachkunde, seine durchdachten, abgewogenen und oft originellen Argumente, sein waches Interesse an fremden Standpunkten und seine anschaulichen Darstellungen technischer und nicht-technischer Zusammenhänge machten ihn jedoch zu einem überzeugungsstarken Gesprächspartner.

Unser Mitgefühl ist bei seiner Familie, seinen Freunden, seinen Kollegen, seinen Mitarbeitern und seinen Studenten. Er wird ihnen und uns fehlen. Der Datenschutz ist mit seinem Tod um ein Stück Gewissen ärmer.

Für die Herausgeber und den Beirat der DuD

Dirk Fox, Helmut Reimer 